\title{
Object Recognition using Still Image
}

\author{
Deepika T.V ${ }^{1}$, Stafford Michahial ${ }^{1}$, Dr M Shivakumar ${ }^{2}$ \\ Asst Prof, Dept. of E\&IE, GSSSIETW ${ }^{1}$ \\ Prof \& Head, Dept. of E\&IE, GSSSIETW ${ }^{2}$
}

\begin{abstract}
Detection of objects in cluttered scenes is a fundamental challenge that has only recently been widely undertaken by computer vision systems. This paper proposes a novel method how to detect a particular object in cluttered scenes, given a reference image of the object. This paper presents an algorithm for detecting a specific object based on finding point correspondences between the reference and the target image. It can detect objects despite a scale change or in-plane rotation. It is also robust to small amount of out-of-plane rotation and conclusion.
\end{abstract}

Keyword: Point Feature Matching, Detection of Features, Cluttered Scenes, Computer Vision Systems.

\section{INTRODUCTION}

Vision systems [14] are increasingly used in the fields of industrial automation and home robotics. Real-time object learning and detection are important and challenging tasks in Computer Vision. Among the application fields that drive development in this area, robotics especially has a strong need for computationally [13][8] efficient approaches, as autonomous systems continuously have to adapt to changing and unknown environment, and to learn and recognize new objects. For such time-critical applications, point feature matching is an attractive solution because new objects can be easily learned online, in contrast to statistical-learning techniques that require many training samples. Our approach is related to recent and efficient matching methods and more particularly to, which consider only images and their gradients to detect objects. The method of object detection works best for objects that exhibit non-repeating texture patterns, which give rise to unique feature matches. This technique is not likely to work well for uniformly-coloured objects, or for objects containing repeating patterns. The proposed algorithm is designed for detecting a specific object. The method of object detection works best for objects that exhibit non-repeating texture patterns, which give rise to unique feature matches[2]. This technique is not likely to work well for uniformly coloured objects, or for objects containing repeating patterns. The proposed algorithm is designed for detecting a specific object, for example, the elephant in the reference image, rather than any elephant. For detecting objects of a particular category, such as people or faces etc, The structure of the paper is as follows: Section II gives an algorithm Implementation. Section III gives simulation results. Section IV gives conclusion.

\section{METHODOLOGY}

The fig.1 shows the basic steps involved in the proposed algorithm implementation. Firstly, Read the reference image containing the object of interest and target image containing a cluttered scene.

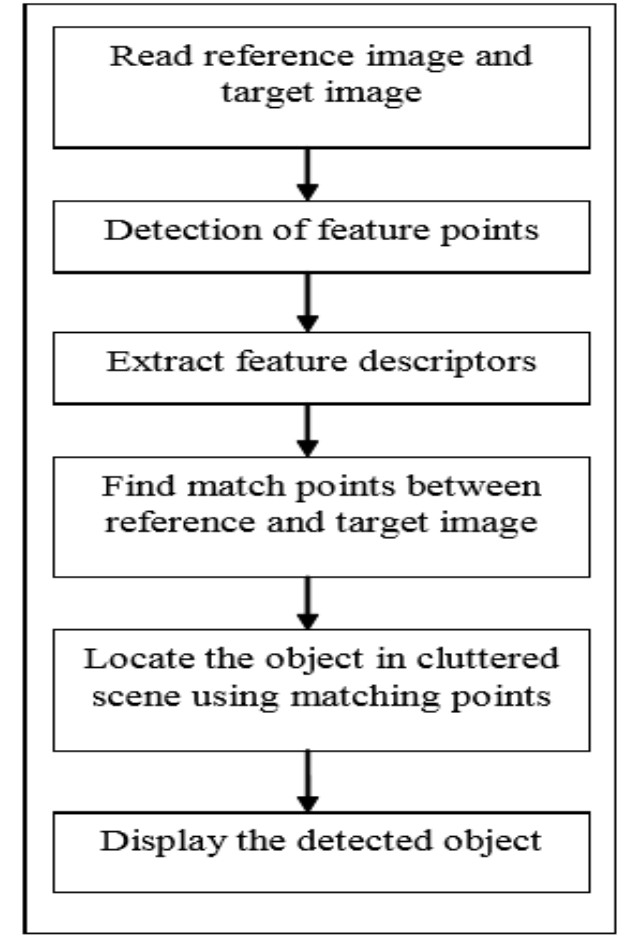

Fig. 1: Algorithm for Detection of Objects in Cluttered Scene

After reading reference and target image perform feature detection process on both images. Feature detection is the process where we automatically examine an image to extract features, which are unique to the objects in the image, in such a manner that we are able to detect an object based on its features in different images.

The processes of feature detection can be divided in to 3 steps.

\section{A. Detection}

Automatically identify interesting features, interest points this must be done robustly. The same feature should always be detected regardless of viewpoint 
Vol. 5, Issue 12, December 2016

\section{B. Description}

Each interest point should have a unique description that does not depend on the features scale and rotation.[4]

\section{Matching}

Given input image, determine which objects it contains, and possibly a transformation of the object, based on predetermined interest points. In this paper, we are use SURF9(Speeded Up Robust Features) algorithm to detect features because of it should provide better results, faster than SIFT algorithm. SURF uses a hessian based blob detector to find interest points.The determinant of a hessian matrix expresses the extent of the response and is an expression of the local change around the area.[15]

The heart of the SURF detection is non-maximalsuppression of the determinants of the hessian matrices. The convolution is very costly to calculate and it is approximated and speeded-up with the use of integral images and approximated kernels. To detect features across scale we have to examine several octaves and levels, where SIFT scales the image down for each octave and use progressively larger Gaussian kernels, the integral images allows the SURF algorithm[1] to calculate the responses with arbitrary large kernels. The purpose of a descriptor is to provide a unique and robust description of a feature; a descriptor can be generated based on the area surrounding an interest point. The SURF descriptor is based on Haar wavelet responses and can be calculated efficiently with integral images. SIFT uses another scheme for descriptors based on the Hough transforms. Common to both schemes is the need to determine the orientation. By determining a unique orientation for an interest point, it is possible to achieve rotational invariance. Before the descriptor is calculated the interest area surrounding the interest point are rotated to its direction.

The SURF descriptors are robust to rotations and an upright version, U-SURF, should be robust for rotations \pm 15 degrees without performing an orientation assignment. I have implemented the upright version, and will not go into further detail on orientation assignment. Next, match the features including outliers using their descriptors. Finally locate the object in scene using matched points. In order to locate the object in scene estimate geometric transformations i.e. affine transform. Estimate Geometric Transform calculates the transformation relating the matched points, while eliminating outliers. This transformation allows us to localize the object in the scene and finally transform the reference image into the coordinate system of the target image[11]. The transformed image indicates the location of the object in the scene.

\section{RESULTS AND DISCUSSION}

The method of object detection[3] works best for objects that exhibit non-repeating texture patterns, which give rise to unique feature matches. The proposed algorithm is for detecting a specific object based on finding point correspondences between the reference and the target image. This paper proposes a novel method how to detect a particular object in cluttered scene, given a reference image of the object.

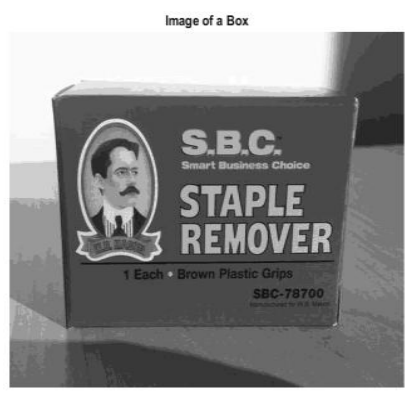

Fig.2:Reference image

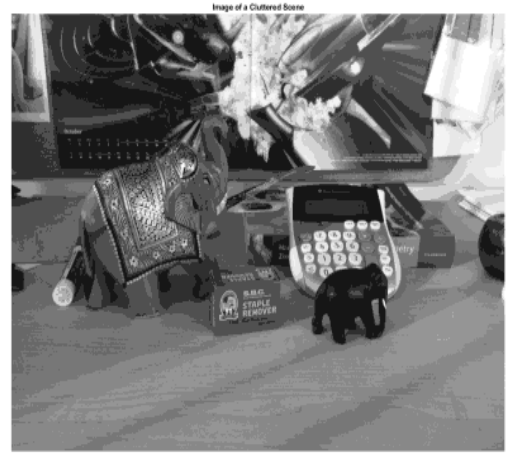

Fig.3:cluttered scene image

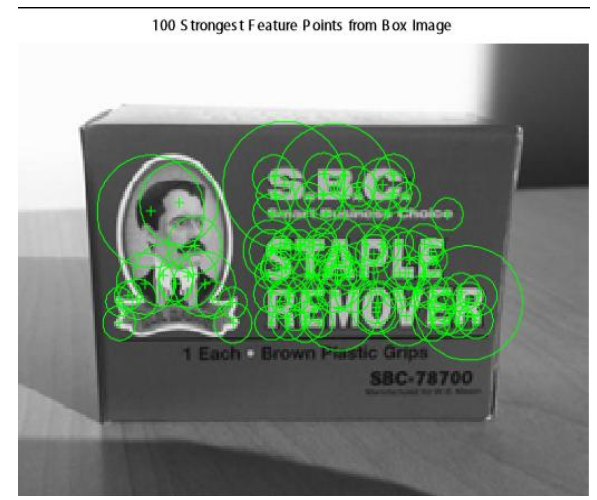

Fig.4: feature points from reference image

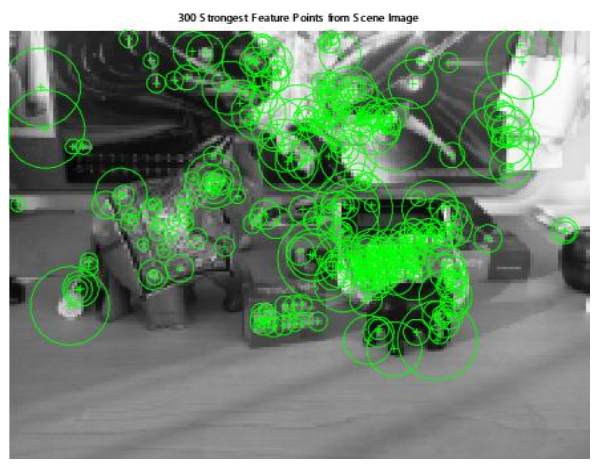

Fig.5: feature points from cluttered scene image 


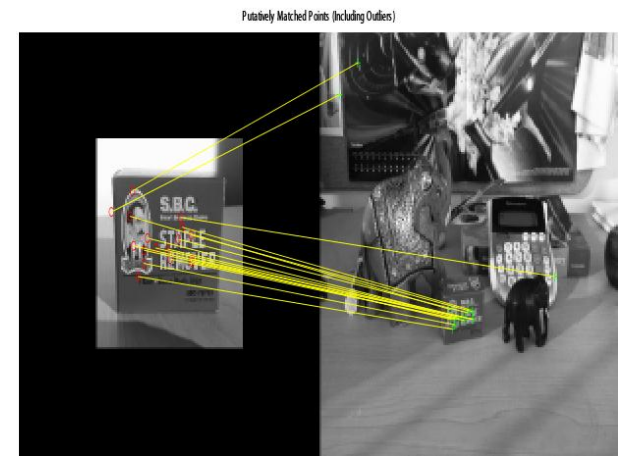

Fig.6:matching points including outliers

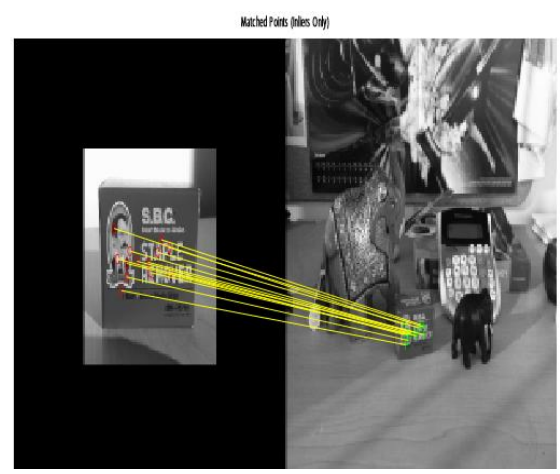

Fig.7:Matching points excluding outliers

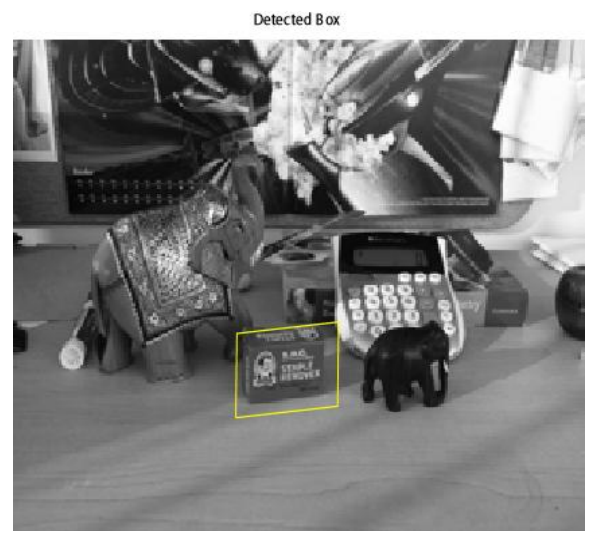

Fig. 8: Detected Object

\section{CONCLUSION}

The proposed algorithm is for detecting a specific object based on finding point correspondences between the reference and the target image. It can detect objects despite a scale change or in plane rotation. It is also robust to small amount of out-of-plane rotation and occlusion. This method of object recognition[12] works best for objects that exhibit non-repeating texture patterns, which give rise to unique feature matches. This technique is not likely to work well for uniformly-colored objects, or for objects containing repeating patterns.

\section{REFERENCES}

[1] S. U. R. F. (SURF), "Herbet bay, andreas ess, tinne tuytelaars, luc van gool", Elsevier preprint, 2008.
[2] University of Koblenz-Landau, Tech. Rep., 2009.

[3] Wikipedia, "Feature detection Wikipedia, the free encyclopedia", 2011

[4] Jacob Toft Pedersen,"Study group SURF: Feature detection \& description".

[5] Chen et al. A moving object tracked by a mobile robot with real time obstacles avoidance capacity. In International Conference on pattern recognition, 2006.

[6] Gohring et al.Multi Robot Object Tracking and Self Localization using Visual percept relations. In International Conference on intelligent robots and systems, 2006.

[7] Kobilarov et al. People Tracking and Following with Mobile Robot Using and Omni directional Camera and Laser. In International Conference on Robotics and Automation, 2006

[8] $\mathrm{Li}$ et al. Computation offloading to save energy on Handled devices: A partial scheme. In International Conference on compilers, Architecture and synthesis for embedded system, 2001.

[9] Nurmi et al. The Eucalyptus Open-Source Cloud-Computing System. In International symposium on cluster computing and the grid, pages 124-131.IEEE computer society, 2009.

[10] Powell et al. Process migration in DEMOS/MP.ACM SIGOPS Operating systems review, 17(5):110-119, 1983.

[11] Qian et al. Simultaneous Robot Localization and person tracking using Rao-blackwellised particle filters with multi model sensors. In IEEE/RSJ IROS, 2008.

[12] Serre et al. Object Recognition with Features Inspired by visual cortex. In IEEE CVPR,

[13] Xian et al. Adaptive Computation Offloading for Energy Conservation on Battery-Powered Systems. In ICPDS, 2007.

[14] David Lowe. Object Recognition from Local Scale-Invariant Features. In International Conference on computer vision, 1999.

[15] M.Piccardi. Background Subtraction Techniques: A Review. In International Conference on systems, Man and Cybernetics, 2004. 